\title{
Enumeration algorithms for FDH directional distance functions under different returns to scale assumptions*
}

\author{
Kristiaan Kerstens ${ }^{\dagger} \quad$ Ignace Van de Woestyne ${ }^{\ddagger}$ \\ Original version: 24th February 2017 \\ This version: 12th December 2017
}

\begin{abstract}
Computing directional distance functions for a free disposal hull (FDH) technology in general requires solving nonlinear mixed integer programs. Cherchye, Kuosmanen, and Post (2001) provide an enumeration algorithm for the FDH directional distance function in case of a variable returns to scale (VRS) technology. In this contribution, we provide fast enumeration algorithms for the FDH directional distance functions under constant (CRS), nonincreasing (NIRS), and nondecreasing (NDRS) returns to scale assumptions. Consequently, enumeration algorithms are now available for all commonly used returns to scale assumptions.
\end{abstract}

Keywords: directional distance function, enumeration, free disposal hull, CRS, NDRS, NIRS

\section{Introduction}

Traditionally, convexity of technology is a maintained axiom in the nonparametric approach to production theory (see Afriat (1972), Charnes, Cooper, and Rhodes (1978) or Diewert and Parkan (1983)). ${ }^{1}$ Nevertheless, Afriat (1972) was probably the first to mention a basic

\footnotetext{
${ }^{*}$ We acknowledge helpful comments of three most constructive referees. The usual disclaimer applies.

${ }^{\dagger}$ CNRS-LEM (UMR 9221), IESEG School of Management, 3 rue de la Digue, F-59000 Lille, France. k. kerstens@ieseg.fr, Corresponding author.

${ }^{\ddagger}$ KU Leuven, Research unit MEES, Warmoesberg 26, B-1000 Brussel, Belgium.

${ }^{1}$ One often uses the moniker Data Envelopment Analysis (DEA) when imposing convexity on technology.
} 
nonconvex Free Disposal Hull (FDH) model imposing the assumptions of strong disposal of inputs and outputs solely. This single output specification has been generalized to the multiple output case in Deprins, Simar, and Tulkens (1984). While computing radial efficiency measures relative to convex technologies normally requires solving a linear programming problem (LP) for each evaluated observation, this becomes a binary mixed integer programming problem (BIP) for the nonconvex FDH model. The use of enumeration for this basic FDH model has been around in the literature since a while (see, e.g., Deprins, Simar, and Tulkens (1984), Fried, Lovell, and Turner (1996), Tulkens (1993)).

Though it is clear that this nonconvex FDH technology is nowhere as popular as its convex counterpart, a rather substantial amount of studies have adopted this simple model. A selection of empirical examples oriented towards some US sectors include: Alam and Sickles (2000) explore the dynamics of technical efficiency in the deregulated airline industry; Fried, Lovell, and Vanden Eeckaut (1993) assess the performance of credit unions; Walden and Tomberlin (2010) provides convex and nonconvex plant capacity estimates in fisheries; etc.

An important extension on this basic FDH model has been proposed in Kerstens and Vanden Eeckaut (1999) when specific returns to scale assumptions have been introduced and a new goodness-of-fit method is defined to characterize returns to scale for nonconvex technologies. Computing radial efficiency measures relative to these extended FDH technologies now requires solving a nonlinear binary mixed integer program (NLBIP). This initial state of affairs triggered three distinctive solution strategies aimed at reducing this computational complexity. First, Podinovski (2004) reformulates these nonconvex extended FDH technologies as binary mixed integer programs (BIP) using a big M technique. Second, starting from an existing LP model for the basic FDH model (see Agrell and Tind (2001)), Leleu (2006) manages to formulate a series of equivalent LP problems. Third, Briec, Kerstens, and Vanden Eeckaut (2004) propose an implicit enumeration strategy for these nonconvex extended FDH technologies to obtain closed form solutions for the radial input efficiency measure. Briec and Kerstens (2006) refine this analysis somewhat by developing enumeration also for radial output- and graph-oriented efficiency measures, and furthermore indicate that the computational complexity of enumeration is advantageous compared to all previous proposals. Kerstens and Van de Woestyne (2014b) offer an empirical perspective on this computational complexity issue and obtain that enumeration is by far the fastest approach, but that LP is even slower than BIP because of the sheer size of the Leleu (2006) formulation.

Empirical examples of applications of these extended FDH technologies include: Cesaroni (2011) computes returns to scale for the case of the Italian driver and vehicle agency; Destefanis and Sena (2005) investigate productivity change using an intersection of these 
nonconvex technologies; De Witte and Marques (2011) determine optimal scale size for the Portuguese drinking water sector; Mairesse and Vanden Eeckaut (2002) evaluate returns to scale for museums in the Walloon region of Belgium²; etc.

Kerstens and Van de Woestyne (2014b, p. 10) mention that an obvious advantage of the LP approach is that it is always available as an option. If a practitioner needs a specialized version of these basic nonconvex production models (for example, a sub-vector radial input efficiency measure defined on some of the input dimensions solely), then these needs are unlikely covered in some of the available software packages (see Barr (2004) for an overview). Under these circumstances, it may be easier to program an LP in a standard optimization software (e.g., Green (1996) or Olesen and Petersen (1996)) rather than derive the required implicit enumeration algorithm. In order to neutralize this convenience advantage of LP, one would need to come up with a general formulation of these implicit enumeration algorithms covering a wide variety of special production models (e.g., apart from different returns to scale, also different measurement orientations of efficiency, different sub-vector cases, etc). Such general formulation is currently lacking.

The purpose of this note is to come up with the broadest possible formulation of an implicit enumeration algorithm for extended FDH technologies by focusing on the most general efficiency measure available in the literature. With the introduction of the shortage function, Luenberger (1992a; 1992b) has managed to generalize all existing distance functions and provides a flexible tool to account for both input contractions and output improvements when measuring efficiency. Luenberger (1992b; 1995) and Chambers, Chung, and Färe (1998) show that the shortage function (or directional distance function in the terminology of Chambers, Chung, and Färe (1998)) is dual to the profit function. While Cherchye, Kuosmanen, and Post (2001) develop an enumeration algorithm for the general directional distance function when assuming a basic FDH technology, nobody ever developed the algorithm for extended FDH technologies. Therefore, we offer an implicit enumeration algorithm for the directional distance function for the extended FDH technologies.

Section 2 defines the extended FDH technologies as well as the directional distance function. It also contains the key proposition with the enumeration algorithm for the general directional distance function for the extended FDH technologies. Two remarks treat, amongst others, the special cases that can be deduced. Section 3 contains an empirical illustration contrasting computational times for enumeration versus LP.

\footnotetext{
${ }^{2}$ These same authors also innovate methodologically by adding lower and upper bound restrictions to scaling in these extended FDH models.
} 


\section{Nonparametric technologies and directional distance functions}

\subsection{Basic definitions}

In a production context, inputs $x \in \mathbb{R}_{+}^{p}$ are transformed to outputs $y \in \mathbb{R}_{+}^{q}$. The set $T=\left\{(x, y) \in \mathbb{R}_{+}^{p} \times \mathbb{R}_{+}^{q} \mid x\right.$ can produce $\left.y\right\}$ of all possible input-output combinations is referred to as the corresponding technology.

Consider available $n$ observations $\left(x_{1}, y_{1}\right), \ldots,\left(x_{n}, y_{n}\right) \in \mathbb{R}_{+}^{p} \times \mathbb{R}_{+}^{q}$. Let $X=\left(x_{1} \ldots x_{n}\right)^{t} \in$ $\mathbb{R}^{n \times p}$ and $Y=\left(y_{1} \ldots y_{n}\right)^{t} \in \mathbb{R}^{n \times q}$ denote the matrices holding all inputs and outputs of these observations, with the superscript $t$ referring to the transposition operation on matrices. Elements of these input and output matrices are referred to by a double index indicating the row and column number of occurrence. For example, $X_{i, j}$ denotes the $j$-th input for the $i$-th observation and $Y_{i, k}$ denotes the $k$-th output for this same $i$-th observation. Furthermore, $x_{j}$ represents the $j$-th input for a given observation, and $y_{k}$ represents the $k$-th output for a given observation.

Following the unified nonparametric approach of Briec, Kerstens, and Vanden Eeckaut (2004), technology can then be represented algebraically by

$$
T^{\Lambda, \Gamma}=\left\{(x, y) \in \mathbb{R}_{+}^{p} \times \mathbb{R}_{+}^{q} \mid \delta z X \leq x, \delta z Y \geq y, z \in \Lambda, \delta \in \Gamma\right\}
$$

with

(i) $\Gamma=\{\delta \in \mathbb{R} \mid \delta \geq 0\}$ in the case of constant returns to scale (CRS),

(ii) $\Gamma=\{\delta \in \mathbb{R} \mid 0 \leq \delta \leq 1\}$ in the case of nonincreasing returns to scale (NIRS),

(iii) $\Gamma=\{\delta \in \mathbb{R} \mid \delta \geq 1\}$ in the case of nondecreasing returns to scale (NDRS),

(iv) $\Gamma=\{\delta \in \mathbb{R} \mid \delta=1\}$ in the case of variable returns to scale (VRS),

$\Lambda=\left\{z \in \mathbb{R}^{n} \mid \forall i \in\{1, \ldots, n\}: z \mathbf{1}=1, z_{i} \in \mathbb{R}_{+}\right\}$if convexity is assumed, or $\Lambda=\left\{z \in \mathbb{R}^{n} \mid\right.$ $\left.\forall i \in\{1, \ldots, n\}: z \mathbf{1}=1, z_{i} \in\{0,1\}\right\}$ if nonconvexity is assumed, and $\mathbf{1}=(1 \ldots 1)^{t} \in \mathbb{R}^{n}$.

For a general technology $T$, the directional distance function $D_{T}: T \times\left(\mathbb{R}_{+}^{p} \times \mathbb{R}_{+}^{q}\right) \rightarrow$ $\mathbb{R} \cup\{+\infty\}$ is defined by $D_{T}\left(x, y ; g_{x}, g_{y}\right)=\sup _{\theta}\left\{\theta \in \mathbb{R} \mid\left(x-\theta g_{x}, y+\theta g_{y}\right) \in T\right\} .{ }^{3}$ Applied

\footnotetext{
${ }^{3}$ Note that the directional distance function is more general than the graph-oriented efficiency measure
} 
to the nonparametric technology $T^{\Gamma, \Lambda}$ defined in (1), this directional distance function now becomes

$$
D_{T}\left(x, y ; g_{x}, g_{y}\right)=\max _{\theta, z}\left\{\theta \in \mathbb{R} \mid \delta z X \leq x-\theta g_{x}, \delta z Y \geq y+\theta g_{y}, z \mathbf{1}=1, z \in \Lambda, \delta \in \Gamma\right\}
$$

\subsection{Convexity is questionable}

Though the axiom of convexity is traditionally maintained in economics and part of the operations research literature dealing with frontier methods, we develop three types of arguments to question it. One argument is related to economic theory. One argument is pragmatic: in empirical applications managers often object to convexity. A final argument is just empirical: convexity simply matters because it affects the results of cost function estimates, technical efficiency estimates, productivity growth, etc.

First, in economic theory convexity of technology is interpreted solely in terms of the time divisibility of technologies. ${ }^{4}$ For instance, Hackman (2008, p. 39) is clear when discussing the axiom of convexity in his textbook:

It does have the following "time-divisibility" justification. Suppose input vectors $x_{1}$ and $x_{2}$ each achieve output level $u>0$. Pick a $\lambda \in[0,1]$, and imagine operating $100 \lambda \%$ of the time using $x_{1}$ and $100(1-\lambda) \%$ of the time using $x_{2}$. At an aggregate level of detail, it is not unreasonable to assume that the weighted average input vector $\lambda x_{1}+(1-\lambda) x_{2}$ can also achieve output level $u$.

This time divisibility argument ignores setup and lead times that make switching between underlying activities costly in terms of time. Thus, convexity is questionable when time indivisibilities compound all other reasons for spatial nonconvexities (e.g., indivisibilities, increasing returns to scale, economies of specialization, externalities, etc.).

Second, in remarks scattered in the literature, one finds evidence on the problems communicating the results of traditional efficiency measurement assuming convexity to decision makers. For example, Epstein and Henderson (1989, p. 105) reports how managers simply

mentioned above. First, the direction vector can take any values. Second, while the directional distance function is dual to the profit function, a graph-oriented (or hyperbolic) efficiency measure is only dual to the return to the dollar function which measures profitability (see Färe, Grosskopf, and Zaim (2002)).

${ }^{4}$ Sometimes the motivation to maintain convexity is just analytical convenience (see, e.g., Hackman (2008, p. 2)). This is an argument that can hardly be taken seriously. 
question the feasibility of the hypothetical projection points resulting from convex nonparametric frontiers when discussing an application to a large public-sector organisation:

The algorithm for construction of the frontier was also discussed. The frontier segment connecting A and B was considered unattainable. It was suggested that either (1) these two DMUs should be viewed as abnormal and dropped from the model, (2) certain key variables have been excluded, or (3) the assumption of linearity was inappropriate in this organization. It appears that each of these factors was present to some degree.

Finally, in empirical applications nonconvexity matters compared to traditional convex analysis. In particular, we provide some evidence for a selection of four topics: (i) cost functions, (ii) technical efficiency, (iii) productivity growth, and (iv) capacity utilisation.

In a study on Spanish municipalities, Balaguer-Coll, Prior, and Tortosa-Ausina (2007) reveal that convex costs are only $58.87 \%$ of nonconvex costs at the sample average. Analysing the U.S. life insurance industry, Cummins and Zi (1998) even report that convex cost are about half of the nonconvex costs. Analysing Belgian municipalities De Borger and Kerstens (1996) show that convex costs are only $77.59 \%$ of convex costs.

Analysing the world's major container ports and terminals, Cullinane, Song, and Wang (2005) report substantial differences between convex and nonconvex technical efficiency results (no statistical tests are reported). Similarly, evaluating UK economics departments Mayston (2014) finds substantial differences in technical efficiency at the sample level (again no statistical tests are reported).

Kerstens and Van de Woestyne (2014a) report empirical results for the Malmquist productivity index as well as for the Hicks-Moorsteen Total Factor Productivity (TFP) index under various specifications of technology. For both indices, it turns out that convex and nonconvex results for both CRS and VRS yield different descriptive statistics, though no formal tests are provided regarding the statistical significance of these differences. Kerstens and Managi (2012) focus on the Luenberger productivity indicator and find that productivity change is on average significantly smaller under nonconvexity, and that both $\beta$-convergence and $\sigma$-convergence only occur under nonconvexity.

Walden and Tomberlin (2010) report average output-oriented plant capacity estimates that vary between $52 \%$ and $84 \%$ in the cases of basic convex and nonconvex technologies, respectively. Cesaroni, Kerstens, and Van de Woestyne (2017) define an alternative inputoriented plant capacity notion and report numbers of $120 \%$ and $121 \%$ for the convex and 
nonconvex technologies, respectively: these apparent small differences reflect distributions that are statistically significantly different.

The upshot is that convexity is questionable from a theoretical, a pragmatic as well as from an empirical point of view.

\subsection{Computational issues and main results}

Obviously, the optimization model determined by (2) contains nonlinear constraints. Under the assumption of convexity, the optimization model (2) can be straightforwardly transformed into an LP (see, e.g., Briec and Kerstens (2006)). Under nonconvexity, Leleu (2009) provides alternative linear programs (LPs) for computing these directional distance functions. These LPs allow for computing the directional distance function using any of the widely available LP solvers. In the case of FDH, Cherchye, Kuosmanen, and Post (2001) provide an enumeration algorithm for the general directional distance function when assuming a VRS technology. This algorithm outperforms the corresponding LP solution and does not require any high level optimizer. Simple operations such as finding the minimum or maximum of finite lists suffice.

We now determine enumeration algorithms for the FDH directional distance function in the cases of CRS, NIRS and NDRS that - to the best of our knowledge- are missing in the literature. As in the case of VRS, these enumeration algorithms outperform the LP equivalents of Leleu (2009) and only require finding minima and maxima of finite lists.

Proposition 2.1. For an observation $\left(x^{o}, y^{o}\right) \in T$ and a direction $\left(g_{x}, g_{y}\right) \in \mathbb{R}_{+}^{p} \times \mathbb{R}_{+}^{q}$ realizing a feasible FDH directional distance function, this function can be obtained by

$$
D_{T}\left(x^{o}, y^{o} ; g_{x}, g_{y}\right)=\max _{i \in\{1, \ldots, n\}}\left(\min _{\substack{j \in\{1, \ldots, p\} \\ k \in\{1, \ldots, q\}}} \theta_{i, j, k}\right)
$$

with

(a) $\theta_{i, j, k}=\frac{Y_{i, k} x_{j}^{o}-X_{i, j} y_{k}^{o}}{Y_{i, k}\left(g_{x}\right)_{j}+X_{i, j}\left(g_{y}\right)_{k}}$ in the case of $C R S$;

(b) $\theta_{i, j, k}=\left\{\begin{array}{ll}\frac{Y_{i, k} x_{j}^{o}-X_{i, j} y_{k}^{o}}{Y_{i, k}\left(g_{x}\right)_{j}+X_{i, j}\left(g_{y}\right)_{k}} & \text { if } \frac{y_{k}^{o}\left(g_{x}\right)_{j}+x_{j}^{o}\left(g_{y}\right)_{k}}{Y_{i, k}\left(g_{x}\right)_{j}+X_{i, j}\left(g_{y}\right)_{k}} \leq 1 \\ \frac{Y_{i, k}-y_{k}^{o}}{\left(g_{y}\right)_{k}} & \text { else }\end{array}\right.$ in the case of NIRS; 
(c) $\theta_{i, j, k}=\left\{\begin{array}{ll}\frac{Y_{i, k} x_{j}^{o}-X_{i, j} y_{k}^{o}}{Y_{i, k}\left(g_{x}\right)_{j}+X_{i, j}\left(g_{y}\right)_{k}} & \text { if } \frac{y_{k}^{o}\left(g_{x}\right)_{j}+x_{j}^{o}\left(g_{y}\right)_{k}}{Y_{i, k}\left(g_{x}\right)_{j}+X_{i, j}\left(g_{y}\right)_{k}} \geq 1 \\ \frac{x_{j}^{o}-X_{i, j}}{\left(g_{x}\right)_{j}} & \text { else }\end{array}\right.$ in the case of NDRS.

Proof. Since FDH is assumed, the activity vector $z$ in (2) must have binary components summing up to one. Hence, only the $n$ vectors $(1,0, \ldots, 0)$ to $(0, \ldots 0,1)$ of the standard basis of $\mathbb{R}^{n}$ can act as $z$. Consequently, (2) can be rewritten as $D_{T}\left(x^{o}, y^{o} ; g_{x}, g_{y}\right)=\max \left\{\theta_{i} \mid\right.$ $i \in\{1, \ldots, n\}\}$ with

$\theta_{i}=\max \left\{\theta \in \mathbb{R} \mid \delta X_{i, j} \leq x_{j}^{o}-\theta\left(g_{x}\right)_{j}, \delta Y_{i, k} \geq y_{k}^{o}+\theta\left(g_{y}\right)_{k}, \delta \in \Gamma, j \in\{1, \ldots, p\}, k \in\{1, \ldots, q\}\right\}$

(a) Consider the case of CRS. Then $\delta \geq 0$ and for each $i \in\{1, \ldots, n\}$, expression (3) can be seen as an LP problem with $p q$ linear inequality constraints in the two variables $\delta$ and $\theta$. The optimal solution of such an LP must be reached at the intersection of two straight lines corresponding with two of these inequality constraints. But since all input related constraints (i.e., $\left.\delta X_{i, j} \leq x_{j}^{o}-\theta\left(g_{x}\right)_{j}\right)$ have a negative slope while all output related constraints (i.e., $\delta Y_{i, k} \geq y_{k}^{o}+\theta\left(g_{y}\right)_{k}$ ) have a positive slope, the optimal solution $\theta_{i}$ can only be found at the intersection of an input and an output related straight line. Indeed, assume the optimal solution $\theta_{i}$ is found at the intersection of two input related straight lines. Then, given the negative slopes of both straight lines, further improvement of $\theta_{i}$ must be possible which contradicts the optimality assumption. The same reasoning can be applied to the combination of two output related straight lines.

Cramer's rule for solving the system of linear equation when combining the $j$-th input constraint with the $k$-th output constraint leads to the solution

$$
\delta_{i, j, k}=\frac{y_{k}^{o}\left(g_{x}\right)_{j}+x_{j}^{o}\left(g_{y}\right)_{k}}{Y_{i, k}\left(g_{x}\right)_{j}+X_{i, j}\left(g_{y}\right)_{k}} \text { and } \theta_{i, j, k}=\frac{Y_{i, k} x_{j}^{o}-X_{i, j} y_{k}^{o}}{Y_{i, k}\left(g_{x}\right)_{j}+X_{i, j}\left(g_{y}\right)_{k}} \text {. }
$$

Obviously, from the basic assumptions, $\delta_{i, j, k} \geq 0$ which is needed in the case of CRS. By taking $\theta_{i}=\min \left\{\theta_{i, j, k} \mid j \in\{1, \ldots, p\}\right.$ and $\left.k \in\{1, \ldots, q\}\right\}$, it is guaranteed that all inequality constraints in (3) are satisfied simultaneously hence leading to the requested result.

(b) Assuming NIRS, the additional constraint $\delta \leq 1$ needs to be added to constraints already present in (3). As in case (a), the optimal solution $\theta_{i}$ of (3) is realized by intersecting two corresponding straight lines. Combining the $j$-th input and $k$-th output constraint leads to the same solution $\delta_{i, j, k}$ and $\theta_{i, j, k}$ as the one presented in (4). If $\delta_{i, j, k} \leq 1$, then the combination with the corresponding $\theta_{i, j, k}$ is feasible. However, if $\delta_{i, j, k}>1$, then the 
combination with the corresponding $\theta_{i, j, k}$ is not feasible. Consequently, the input related constraint leading to the infeasibility should now be replaced by the limiting constraint $\delta \leq 1$. The intersection of the remaining output related constraint and this limiting constraint then yields

$$
\delta_{i, j, k}=1 \text { and } \theta_{i, j, k}=\frac{Y_{i, k}-y_{k}^{o}}{\left(g_{y}\right)_{k}} .
$$

Note that, indeed, the input related constraint should be replaced with $\delta \leq 1$ and not the output related constraint. Assuming the latter, then the intersection of the remaining input related constraint with the limiting constraint $\delta \leq 1$ can impossibly yield the optimal solution $\theta_{i}$ since further improvements can be realized due to the negative slope of the input related constraint. Hence, we obtain the desired result.

(c) If NDRS is assumed, the constraint $\delta \geq 1$ needs to be included to constraints already present in (3). Again as in case (a), the optimal solution $\theta_{i}$ of (3) is the result of intersecting two corresponding straight lines. Combining the $j$-th input and $k$-th output constraint realizes the same solution $\delta_{i, j, k}$ and $\theta_{i, j, k}$ reported in (4). If $\delta_{i, j, k} \geq 1$, then the combination with the corresponding $\theta_{i, j, k}$ is feasible. However, if $\delta_{i, j, k}<1$, then the combination with the corresponding $\theta_{i, j, k}$ is not feasible. Therefore, the output related constraint leading to the infeasibility should be replaced by the limiting constraint $\delta \geq 1$. The intersection of the remaining input related constraint and this limiting constraint now yields

$$
\delta_{i, j, k}=1 \text { and } \theta_{i, j, k}=\frac{x_{j}^{o}-X_{i, j}}{\left(g_{x}\right)_{j}} .
$$

Similar to (b), note that, indeed, the output related constraint should be replaced with $\delta \leq 1$ and not the input related constraint. Assuming the latter, then the intersection of the remaining output related constraint with the limiting constraint $\delta \geq 1$ cannot realize the optimal solution $\theta_{i}$ since further improvements are possible due to the positive slope of the output related constraint. The desired result now follows directly.

Since it is our explicit intention with this most general formulation of the implicit enumeration algorithm for extended FDH technologies to contribute to neutralise what we have called the convenience advantage of LP in Section 1, we add the following remarks.

Remark 2.1. Note that Proposition 2.1 even holds true if for some $i, j, k$ combination, the denominators occurring in the expression of $\theta_{i, j, k}$ equal zero. In these cases, simply set $\theta_{i, j, k}=-\infty$. This negative value will eventually be driven out by other positive values when taking the final maximum. 
Remark 2.2. Proposition 2.1 not only completes the list of enumeration algorithms for computing efficiencies in the case of FDH, it also unifies several of these separate algorithms to a single algorithm. Obviously, this unification simplifies a practical implementation. The following list provides some commonly used choices: ${ }^{5}$

- The radial input efficiency measure for $\left(x^{o}, y^{o}\right) \in T$ equals

$$
D F_{i}\left(x^{o}, y^{o}\right)=1-D_{T}\left(x^{o}, y^{o} ; x^{o}, 0\right) .
$$

- The radial output efficiency measure for $\left(x^{o}, y^{o}\right) \in T$ equals

$$
D F_{o}\left(x^{o}, y^{o}\right)=1+D_{T}\left(x^{o}, y^{o} ; 0, y^{o}\right)
$$

- Denote by $S^{v}$ and $S^{f}=\{1, \ldots, p\} \backslash S^{v}$ the index sets containing the indexes referring to the variable and fixed inputs, respectively. Then, the sub-vector radial input efficiency measure (also called the short-run (SR) input efficiency) reducing only the variable inputs determined by $S^{v}$ and leaving the fixed variables determined by $S^{f}$ unchanged, equals

$$
D F_{i}^{S R}\left(x^{o}, y^{o}\right)=1-D_{T}\left(x^{o}, y^{o} ; g_{x}, 0\right)
$$

with

$$
\left(g_{x}\right)_{j}=\left\{\begin{array}{ll}
x_{j}^{o} & \text { if } j \in S^{v} \\
0 & \text { if } j \in S^{f}
\end{array} .\right.
$$

\section{Numerical application}

To test the performance of the algorithm laid out in Proposition 2.1, we provide in Figure 1 an implementation in Python 3.6 by means of the function dirDistEnum. This function assumes the data structures $x$ and $y$ to be of the type NumPy array, hence, allowing the usage of double indexes. Obviously, this function can easily be adapted to using only lists (of lists) instead, therefore, not needing additional Python modules.

[Figure 1 about here]

\footnotetext{
${ }^{5}$ Other measures (e.g., plant capacity utilization measures) can easily be derived from the choices mentioned here.
} 
The function dirDistEnum is self-contained for easy implementation in existing projects. In lines 2 to 4 , the row and column dimensions of the data structures $\mathrm{x}$ and $\mathrm{y}$ are read. Note that these data structures represent the input and output matrices $X$ and $Y$, respectively. Lines 6 to 27 contain the function theta. This function computes $\theta_{i, j, k}$ as determined in Proposition 2.1 depending on the returns to scale set via the input string rts. Note that the case of the denominator being zero is implemented as well by setting the return value to a large negative number (i.e., $-1 \mathrm{E}+10$ ). In lines 29 and 30, the list of size $n$ containing the minima of $\theta_{i, j, k}$ over the indexes $j$ and $k$ is created. Finally, the maximum of this list is returned in line 31.

Clearly, when fixing the input and output dimensions $p$ and $q$, the implementation in Figure 1 has linear time complexity in $n$ if finding the maximum in line 31 is done by a linear search. The time complexity can even be improved by using a binary heap (e.g., a max-heap in this case) rather than a simple list.

As mentioned earlier, following Leleu (2009), the FDH directional distance function can also be computed by means of solving an LP. Obviously, all currently known algorithms for solving LPs have a time complexity higher than linear time complexity (see e.g., Chong and Zak (2001), Eiselt and Sandblom (2007)). Consequently, the enumeration approach outperforms the LP approach.

To further test this enumeration algorithm, we create in Python 3.6 an artificial data set of 2000 observations with 5 inputs producing 3 outputs. ${ }^{6}$ Thus, the overall input matrix $X \in \mathbb{R}^{2000 \times 5}$ and overall output matrix $Y \in \mathbb{R}^{2000 \times 3}$ consist of uniformly distributed random numbers between 0 and 100. From these overall matrices, sub-matrices containing the first $50 i$ rows, with $i \in\{1, \ldots, 40\}$, are taken to represent the technology. This allows inflating the size of the technology in 40 steps of size 50. Considering the first observation $\left(x^{o}, y^{o}\right)$, the FDH directional distance function is then computed in the position dependent direction (i.e., $\left.g=\left(x^{o}, y^{o}\right)\right)$ using both enumeration and the LP approach. The corresponding LPs are solved by means of the GNU Linear Programming Kit (GLPK), linked to Python. For each technology increasing in size, the computations of both FDH directional distance function implementations are timed. The results of this timing are visualized in Figure 2.

[Figure 2 about here]

\footnotetext{
${ }^{6}$ Empirical data sets of this size are rarely publicly available (e.g., in the Journal of Applied Econometrics Data Archive (http://qed.econ.queensu.ca/jae/)) for the purpose of our illustration.
} 
Although the transfer of the LP problem to GLPK demands a certain overhead, especially notice the nonlinear increase in time of the LP approach contrary to the linear increase of the enumeration method. This observation confirms the theoretical comparison made earlier. Figure 2 also shows that the enumeration method can be applied to large data sets in very reasonable times. Note that Python is actually an interpreter. Hence, additional time gains can be achieved by implementing the algorithm with high performance compilers instead.

The convenience advantage of LP, already mentioned in the Section 1, inspired us to come up with a more general formulation of the enumeration approach for extended FDH technologies. While implementing an LP may also require some programming expertise, coding and debugging time depending on the environment being used, one should acknowledge that using enumeration algorithms requires on average programming expertise, coding and

debugging time slightly exceeding the one required from the LP approach. Therefore, the proposed algorithm may in practice be most useful in situations when the set-up time is a relatively unimportant consideration. Examples may include dealing with large data sets or even so-called big data (e.g., see Dulá (2008)), repetitive computations in, e.g., data mining (e.g., see Akçay, Ertek, and Büyüközkan (2012) or Zhu (2010)), coding the algorithm in software intended for distribution, etc. Finally, note that enumeration has also been employed as a preprocessor to estimate traditional convex frontier models using special LP algorithms (for instance, Sueyoshi (1992)).

\section{Conclusion}

The purpose of this note has been to come up with the broadest possible formulation of an implicit enumeration algorithm for extended FDH technologies for the directional distance function, the most general efficiency measure currently around in the literature. A formal proposition establishes a proof for the implicit enumeration algorithm. A remark treats the special cases that can be easily deduced. An empirical illustration has contrasted computational times for implicit enumeration versus the use of traditional LP: implicit enumeration is the clear winner.

\section{References}

Afriat, S. (1972): "Efficiency Estimation of Production Functions," International Economic Review, 13(3), 568-598. 
Agrell, P., And J. Tind (2001): "A Dual Approach to Nonconvex Frontier Models," Journal of Productivity Analysis, 16(2), 129-147.

AkÇAy, A., G. ERtek, And G. BüyükÖZKan (2012): "Analyzing the Solutions of DEA through Information Visualization and Data Mining Techniques: SmartDEA Framework," Expert Systems with Applications, 39(9), 7763-7775.

Alam, I., And R. Sickles (2000): "Time Series Analysis of Deregulatory Dynamics and Technical Efficiency: The Case of the US Airline Industry," International Economic Review, 41(1), 203-218.

Balaguer-Coll, M., D. Prior, And E. Tortosa-Ausina (2007): "On the Determinants of Local Government Performance: A Two-Stage Nonparametric Approach," European Economic Review, 51(2), 425-451.

BARr, R. (2004): "DEA Software Tools and Technology: A State-of-the-Art Survey," in Handbook on Data Envelopment Analysis, ed. by W. Cooper, L. Seiford, and J. Zhu, pp. 539-566. Kluwer, Boston.

Briec, W., And K. Kerstens (2006): "Input, Output and Graph Technical Efficiency Measures on Non-Convex FDH Models with Various Scaling Laws: An Integrated Approach Based upon Implicit Enumeration Algorithms," TOP, 14(1), 135-166.

Briec, W., K. Kerstens, and P. Vanden Eeckaut (2004): "Non-convex Technologies and Cost Functions: Definitions, Duality and Nonparametric Tests of Convexity," Journal of Economics, 81(2), 155-192.

Cesaroni, G. (2011): "A Complete FDH Efficiency Analysis of a Diffused Production Network: The Case of the Italian Driver and Vehicle Agency," International Transactions in Operational Research, 18(2), 205-229.

Cesaroni, G., K. Kerstens, and I. Van de Woestyne (2017): “A New Input-Oriented Plant Capacity Notion: Definition and Empirical Comparison," Pacific Economic Review, $22(4), 720-739$.

Chambers, R., Y. Chung, and R. Färe (1998): "Profit, Directional Distance Functions, and Nerlovian Efficiency," Journal of Optimization Theory and Applications, 98(2), 351364 .

Charnes, A., W. Cooper, and E. Rhodes (1978): "Measuring the Efficiency of Decision Making Units," European Journal of Operational Research, 2(6), 429-444. 
Cherchye, L., T. Kuosmanen, and T. Post (2001): "FDH Directional Distance Functions with an Application to European Commercial Banks," Journal of Productivity Analysis, 15(3), 201-215.

Chong, E., And S. ZAK (2001): Introduction to Optimization. Wiley, New York, 2 edn.

Cullinane, K., D.-W. Song, and T. Wang (2005): "The Application of Mathematical Programming Approaches to Estimating Container Port Production Efficiency," Journal of Productivity Analysis, 24(1), 73-92.

Cummins, D., And H. Zi (1998): "Comparison of Frontier Efficiency Methods: An Application to the U.S. Life Insurance Industry," Journal of Productivity Analysis, 10(2), $131-152$.

De Borger, B., and K. Kerstens (1996): "Cost Efficiency of Belgian Local Governments: A Comparative Analysis of FDH, DEA, and Econometric Approaches," Regional Science and Urban Economics, 26(2), 145-170.

De Witte, K., and R. Marques (2011): "Big and Beautiful? On Non-Parametrically Measuring Scale Economies in Non-Convex Technologies," Journal of Productivity Analysis, 35(3), 213-226.

Deprins, D., L. Simar, and H. Tulkens (1984): "Measuring Labor Efficiency in Post Offices," in The Performance of Public Enterprises: Concepts and Measurements, ed. by M. Marchand, P. Pestieau, and H. Tulkens, pp. 243-268. North Holland, Amsterdam.

Destefanis, S., And V. Sena (2005): "Public Capital and Total Factor Productivity: New Evidence from the Italian Regions, 197098," Regional Studies, 39(5), 603-617.

Diewert, W., and C. Parkan (1983): "Linear Programming Test of Regularity Conditions for Production Functions," in Quantitative Studies on Production and Prices, ed. by W. Eichhorn, K. Neumann, and R. Shephard, pp. 131-158. Physica-Verlag, Würzburg.

Dulá, J. (2008): "A Computational Study of DEA With Massive Data Sets," Computers 83 Operations Research, 35(4), 1191-1203.

Eiselt, H., And C.-L. Sandblom (2007): Linear Programming and Its Applications. Springer, Berlin.

Epstein, M., And J. Henderson (1989): "Data Envelopment Analysis for Managerial Control and Diagnosis," Decision Sciences, 20(1), 90-119. 
Färe, R., S. Grosskopf, and O. Zaim (2002): "Hyperbolic Efficiency and Return to the Dollar," European Journal of Operational Research, 136(3), 671-679.

Fried, H., C. Lovell, and J. Turner (1996): "An Analysis of the Performance of University Affiliated Credit Unions," Computers \& Operations Research, 23(4), 375-384.

Fried, H., C. Lovell, and P. Vanden Eeckaut (1993): "Evaluating the Performance of U.S. Credit Unions," Journal of Banking \& Finance, 17(2-3), 251-265.

Green, R. (1996): "DIY DEA: Implementing Data Envelopment Analysis in the Mathematical Programming Language AMPL," Omega, 24(4), 489-494.

Hackman, S. (2008): Production Economics: Integrating the Microeconomic and Engineering Perspectives. Springer, Berlin.

Kerstens, K., and S. Managi (2012): "Total Factor Productivity Growth and Convergence in the Petroleum Industry: Empirical Analysis Testing for Convexity," International Journal of Production Economics, 139(1), 196-206.

Kerstens, K., and I. Van de Woestyne (2014a): "Comparing Malmquist and HicksMoorsteen Productivity Indices: Exploring the Impact of Unbalanced vs. Balanced Panel Data," European Journal of Operational Research, 233(3), 749-758.

—_ (2014b): "Solution Methods for Nonconvex Free Disposal Hull Models: A Review and Some Critical Comments," Asia-Pacific Journal of Operational Research, 31(1).

Kerstens, K., and P. Vanden Eeckaut (1999): "Estimating Returns to Scale Using Nonparametric Deterministic Technologies: A New Method Based on Goodness-of-Fit," European Journal of Operational Research, 113(1), 206-214.

Leleu, H. (2006): "A Linear Programming Framework for Free Disposal Hull Technologies and Cost Functions: Primal and Dual Models," European Journal of Operational Research, 168(2), 340-344.

LELEu, H. (2009): "Mixing DEA and FDH models together," Journal of the Operational Research Society, 60(1), 1730-1737.

Luenberger, D. (1992a): "Benefit Function and Duality," Journal of Mathematical Economics, 21(5), 461-481.

Luenberger, D. (1992b): "New Optimality Principles for Economic Efficiency and Equilibrium," Journal of Optimization Theory and Applications, 75(2), 221-264. 
- (1995): Microeconomic Theory. McGraw-Hill, Boston.

Mairesse, F., And P. VAnden Eeckaut (2002): "Museum Assessment and FDH Technology: Towards a Global Approach," Journal of Cultural Economics, 26(4), 261-286.

Mayston, D. (2014): "Effectiveness Analysis of Quality Achievements for University Departments of Economics," Applied Economics, 46(31), 3788-3797.

Olesen, O., And N. Petersen (1996): "A Presentation of GAMS for DEA," Computers $\&$ Operations Research, 23(4), 323-339.

Podinovski, V. (2004): "On the Linearisation of Reference Technologies for Testing Returns to Scale in FDH Models," European Journal of Operational Research, 152(3), 800802.

Sueyoshi, T. (1992): "Measuring Technical, Allocative and Overall Efficiencies using a DEA Algorithm," Journal of the Operational Research Society, 43(2), 141-155.

Tulkens, H. (1993): "On FDH Efficiency Analysis: Some Methodological Issues and Applications to Retail Banking, Courts, and Urban Transit," Journal of Productivity Analysis, 4(1-2), 183-210.

Walden, J., and D. Tomberlin (2010): "Estimating Fishing Vessel Capacity: A Comparison of Nonparametric Frontier Approaches," Marine Resource Economics, 25(1), 23-36.

Zhu, D. (2010): “A Hybrid Approach for Efficient Ensembles," Decision Support Systems, $48(3), 480-487$. 


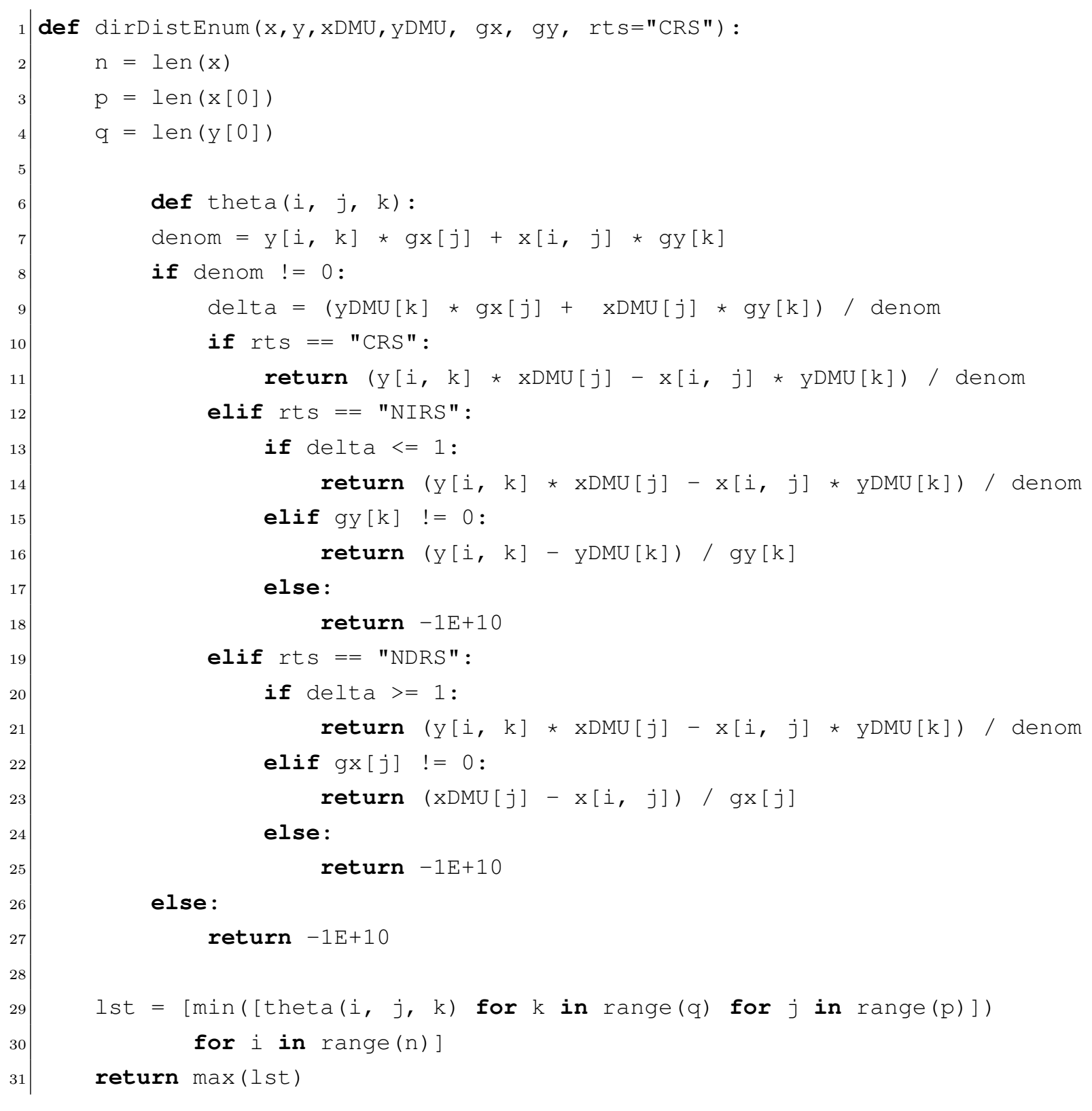

Figure 1: Python 3.6 implementation of the enumeration algorithm suggested in Proposition 2.1 . 


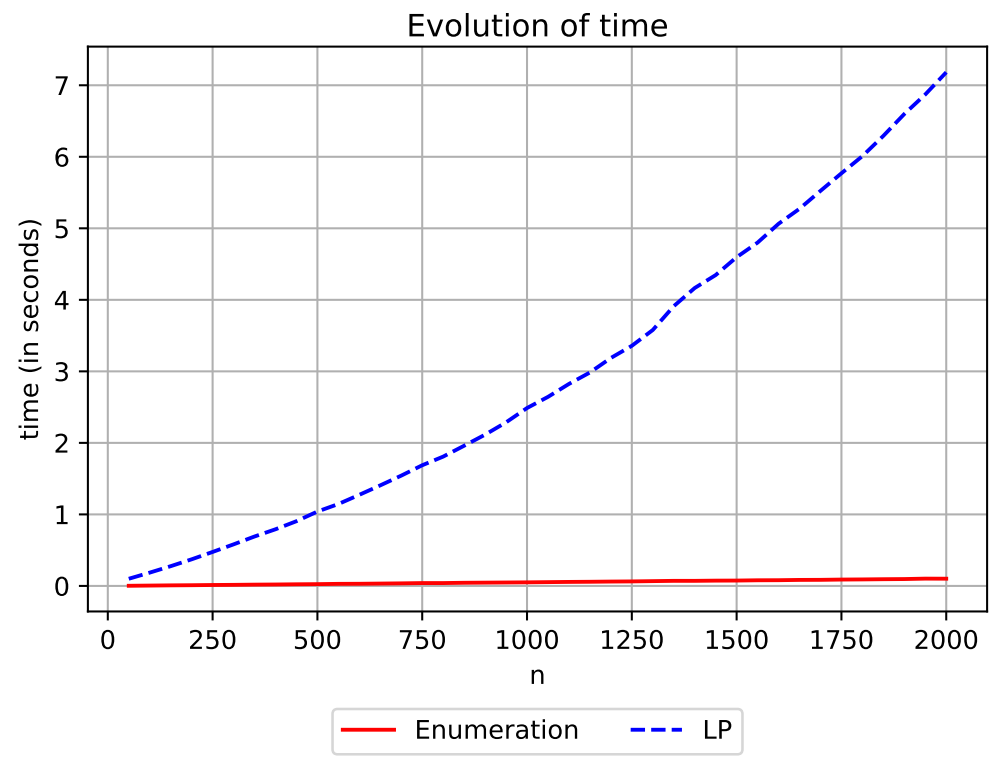

Figure 2: Evolution of time for computing the FDH directional distance function of one observation in relation to the total number of observations $n$ in the technology. 